\title{
Statistical Trends in Family Medicine Journals
}

\section{Hae-Jin Kwon, Yong-Gyu Park*}

Department of Biostatistics, The Catholic University of Korea School of Medicine, Seoul, Korea

Background: This study proposed a desirable direction for the future development of the Korean Journal of Family Medicine (KJFM) by comparing with the overseas SCI journals, Family Medicine (FM) and The Journal of Family Practice (JFP) based on the statistical viewpoints.

Methods: All of the original articles published in KJFM from January 1981 to June 2011, FM from January 1998 to June 2011, and JFP from January 1978 to June 2011, were reviewed and compared in terms of content, data size, research design, and statistical method.

Results: Of 3,226 total original articles, KJFM published 1,549, FM 322, and JFP 1,355, respectively. Both JFP and KJFM mainly focused on biomedical topics ( $67.2 \%$ and $61.7 \%$ ), while FM focused on education (55.9\%). Most of the studies in three journals used the data size of between 100 to 300 cases. The most frequently used research design was cross-sectional, FM 66.8\%, JFP $58.4 \%$, and KJFM 72.4\%, respectively. The statistical methods in KJFM were gradually diversified.

Conclusion: The quality of the original articles in KJFM has been improved over the years, but still has conducted based on the relatively weak research designs. Under the circumstances that the higher ranked SCI journals demand the prospective design and large size of data, and most researchers in Korea could not use the large scaled prospective data, we need to collaborate to accumulate the small sized data sets and try to make a registry. More refined statistical method such as a propensity score matching analysis for retrospective data could be an alternative.

Keywords: Family Medicine Journals; Content Area; Data Size; Research Design; Statistical Method; Statistical Trend

\section{INTRODUCTION}

Korean Journal of Family Medicine (KJFM) is a measure to evaluate the academic results of family medicine in Korea and

Received: November 29, 2011, Accepted: January 16, 2012

*Corresponding Author: Yong-Gyu Park

Tel: +82-2-2258-7226, Fax: +82-2-596-4513

E-mail:ygpark@catholic.ac.kr

Korean Journal of Family Medicine

Copyright (C) 2012 The Korean Academy of Family Medicine

(a) This is an open-access article distributed under the terms of the Creative Commons Attribution Non-Commercial License (http://creativecommons.org/licenses/by-nc/3.0) which permits unrestricted noncommercial use, distribution, and reproduction in any medium, provided the original work is properly cited. plays an important role in leading family medicine. Therefore, it is important to compare the trends of the contents and research designs used in KJFM with those in overseas SCI family medicine journals to understand the current state of KJFM. And a suggestion for a desirable direction also needed to accomplish the academic leap for family medicine.

There have been similar studies on the family medicine journals. Fromm and Snyder ${ }^{1)}$ analyzed the articles in the Journal of Family Practice (JFP) from January 1982 to December 1983 according to research design and statistical method, and concluded that most of the articles used the descriptive statistics and cross-sectional design. In Korea, Yu et al. ${ }^{2)}$ compared there search design, content, quality, and statistical method used in KJFM and JFP from 1981 to 1992, and emphasized that the 
quality of the articles in KJFM was lower than those in JFP; however they also stated that the quality level of the studies has gradually improved. Seo et al. ${ }^{3)}$ analyzed the research design, way of data collection, and statistical method in KJFM from November 1980 to June 1989, and described that $95.2 \%$ of the original articles used only descriptive statistics, $54.2 \%$ of the original articles did not use any statistical method nor mention about the statistical procedure.

KJFM has reached maturity and has been active in not only in the medical area but also in the fields of education and health

Table 1. Category of major content area.

\begin{tabular}{|c|c|c|}
\hline Category & Content & Content detail \\
\hline \multirow[t]{7}{*}{ Biomedical } & Common medical problem & Overall medical problem of patient's disease \\
\hline & Geriatrics & Diagnosis and treatment of disease Affecting older people \\
\hline & Epidemiology & Occurrence, transmission and control of epidemic disease \\
\hline & Procedure & Surgical procedure \\
\hline & Laboratory medicine & It is to get information about the health of a patient as clinical tests for medical purposes \\
\hline & Smoking & Pain and disease related to smoke \\
\hline & Obesity & Pain and disease related to obesity \\
\hline \multirow[t]{9}{*}{ Biopsychosocial } & Stress & Pain and disease related to stress \\
\hline & Doctor-patient relationship & Psychological relationship between doctor and patient \\
\hline & Death and dying & Worry and illness of the mind about death \\
\hline & Psychotherapy & Psychological methods in treating people who are mentally ill \\
\hline & Chemical dependency & It is hard to control, even so it must be controlled \\
\hline & Ethics & Moral value of human conduct and of the rules and principles \\
\hline & Compliance & A patient's adherence to a recommended course of treatment \\
\hline & Family dynamics & Diversity, tradition, love, money, culture, and change related to patient's family \\
\hline & Quality of life & A degree of satisfaction of patient's life \\
\hline \multirow[t]{6}{*}{ Education } & General medical education & Medical education for the general public \\
\hline & Resident education & Medical education for the resident \\
\hline & Undergraduate education & Education of medical student \\
\hline & Faculty development & Education of general student \\
\hline & Research methodology & Details needed to research \\
\hline & Patient education & Medical education for patients \\
\hline \multirow[t]{9}{*}{ Health service } & General service & Common service of medical institution \\
\hline & Health maintenance & Service and try for patient's health maintenance \\
\hline & Cost-effectiveness & Medical expense insurance and overallmedical fee \\
\hline & Clinical decision making & Patient's decision participated to the clinical trial \\
\hline & Continuity of care & Treatment continuity of patient \\
\hline & Quality of care & Quality of medical institution's treatment \\
\hline & Institution management & Overall management of medical institution \\
\hline & Reimbursement & Insurance and medicare reimbursement \\
\hline & Refer and consultation & Doctor and medical team consultation of common disease \\
\hline
\end{tabular}


service. And there is more awareness of the fact that the study of family medicine is a necessary element for the development of family medicine as a professional preliminary medical treatment. Therefore, the need for comparing the KJFM, which reflects 30 years of academic outcomes, with overseas SCI journals of family medicine and providing the proper direction for the future development of KJFM is required.

\section{METHODS}

\section{Subjects}

The subjects of the analysis were 3,226 original articles from three family medicine journals; 1,549 in KJFM from January 1981 to June 2011, 322 in Family Medicine (FM) from January 1998 to June 2011, and 1,355 in JFP from January 1978 to June 2011.

\section{Methods}

Following criteria were used to classify the articles.

1) Content

Referring to the method used when Shank ${ }^{4)}$ categorized research fields of family medicine articles, contents were categorized into biomedical, biopsychosocial, education, and health service as shown in Table 1.

\section{2) Data size}

The data size was classified as under 50, 50-100, 100300, 300-500, 500-1,000, 1,000-3,000, 3,000-5,000, 5,000$10,000,10,000-50,000$, and over 50,000 subjects.

\section{3) Research design}

The research designs were classified as follows.

(1) Cross-sectional studies included studies that used data which observed at a specific time during the disease progress of patients, including health check-ups or National Health and Nutrition Examination Survey (NHANES).

(2) Prospective studies were sub-categorized into clinical trials and cohort studies, including studies in which the outcomes were measured at least twice or repeatedly during a certain period. Cohort studies refer to studies that followed up patients with certain risk factors to find out the results and clinical trials refer to prospective studies that proceed to prove the effects of a treatment.

(3) Retrospective studies were sub-categorized into registry

Table 2. Category of statistical methods.

\begin{tabular}{ll}
\hline \multicolumn{1}{c}{ Statistics } & \multicolumn{1}{c}{ Brief description } \\
\hline No statistical method & No statistical content \\
$\begin{array}{l}\text { Descriptive method } \\
\text { Contingency table }\end{array}$ & Only descriptive analysis \\
T-test & Oni-square test, Fisher's exact test, McNemar's test \\
F-test & ANOVA, ANCOVA, MANOVA, repeated measure ANOVA \\
Correlation analysis & Pearson, Spearman correlation analysis \\
Regression analysis & Simple linear, multiple, logistic regression \\
Epidemiologic statistics & Relative risk, odds ratio, sensitivity, specificity, ROC curve, positive-negative rate, predict value, Kappa \\
Non-parametric tests & $\begin{array}{l}\text { Sign test, Wilcoxon signed rank-sum test, Kruskal-Wallis, Mann-Whitney U-test, Cochran-Mantel-Haenzel test, } \\
\text { Cochran-Amitage trend test, Kolmogorov-Smirnov test, Kendall's Tau-b }\end{array}$ \\
Survival analysis & Life table, log-rank test, Cox proportional hazard regression, Kaplan-Meier estimate \\
Factor analysis & Explanatory factor analysis, Confirmatory factor analysis \\
Reliability analysis & Cronbach's alpha \\
\hline Meta analysis & \\
\hline
\end{tabular}

ROC: receiver operating characteristic. 
studies, case-control studies, and historical cohort studies, including studies that reviewed charts by using medical records. Case-control studies refer to studies that retrospectively identify causes of disease by comparing patients and control group.

\section{4) Statistical methods}

The statistical method that Yu et al. ${ }^{2)}$ used to compare KJFM and JFP, was used and categorized as shown in Table 2. FM was excluded because articles published in FM after 1998 were included in this study and there were too many articles in the education area.

\section{Statistical Analyses}

All results were presented as numbers and percentages according to the classified categories.

\section{RESULTS}

\section{Characteristics of Subjects}

Of the 3,226 original articles, the majority of them were published by KJFM 1,549; followed by JFP 1,355; and then FM 322. Because JFP has been mostly publishing conference papers since 2002, the number of original articles has decreased. FM had the least papers because the articles from 1988 were included in the categorization.

\section{Major Content Area of Family Medicine Journals}

In the biomedical area, KJFM and JFP were $61.7 \%$ and

Table 3. Classification of major content area.

\begin{tabular}{lccc}
\hline \multicolumn{1}{c}{ Content area } & FM & JFP & KJFM \\
\hline Biomedical & $58(18.0)$ & $910(67.2)$ & $955(61.7)$ \\
Biopsychosocial & $29(9.0)$ & $158(11.7)$ & $175(11.3)$ \\
Education & $180(55.9)$ & $154(11.4)$ & $249(16.1)$ \\
Health service & $55(17.1)$ & $133(9.8)$ & $170(11.0)$ \\
Total & $322(100.0)$ & $1,355(100.0)$ & $1,549(100.0)$ \\
\hline
\end{tabular}

Values are presented as number (\%).

FM: Family Medicine, JFP: Journal of Family Practice, KJFM: Korean Journal of Family Medicine.
67.2\%, respectively, and FM was $18.0 \%$. In the biopsychosocial area, FM was $9.0 \%, \mathrm{KJFM}$ and JFP were $11.3 \%$ and $11.7 \%$. In the education area, KJFM and JFP were $16.1 \%$ and $11.4 \%$, and FM was 55.9\%. In the health service area, FM had the highest proportion with $17.1 \%$, while JFP had $9.8 \%$ and KJFM had $11.0 \%$ (Table 3 ).

\section{Special Features of Data Size}

1) Overall study

In all three journals, the majority of the articles used a data size of 100-300 subjects and their proportions were KJFM $29.1 \%$, JFP 26.1\%, and FM 28.6\%. The proportion of articles that used a data size of 5,000-10,000 subjects was the highest in KJFM 2.3\%, then JFP 1.9\%, and FM 1.6\%. The articles that used the data size of over 50,000 subjects were $0.5 \%$ in KJFM, $0.7 \%$ in JFP, and $1.9 \%$ in FM (Table 4).

\section{2) Population-based studies}

The largest data size of FM was 54,520 subjects of the 1996 \& 1997 National Ambulatory Medical Care Surveys based crosssectional study at the US National Center for Health Statistics in 2002 and the second-largest data size was the NHANES I Epidemiologic Follow-up Study with 6,558 subjects in 2005. JFP

Table 4. Classification of data size.

\begin{tabular}{lccc}
\hline No. of subjects & FM & JFP & KJFM \\
\hline Under 50 & $62(19.3)$ & $89(6.6)$ & $172(11.1)$ \\
$50-100$ & $52(16.1)$ & $305(22.5)$ & $314(20.3)$ \\
$100-300$ & $92(28.6)$ & $354(26.1)$ & $450(29.1)$ \\
$300-500$ & $46(14.3)$ & $77(5.7)$ & $140(9.0)$ \\
$500-1,000$ & $25(7.8)$ & $191(14.1)$ & $184(11.9)$ \\
$1,000-3,000$ & $22(6.8)$ & $180(13.3)$ & $155(10.0)$ \\
$3,000-5,000$ & $5(1.6)$ & $97(7.2)$ & $52(3.4)$ \\
$5,000-10,000$ & $5(1.6)$ & $26(1.9)$ & $36(2.3)$ \\
$10,000-50,000$ & $7(2.2)$ & $27(2.0)$ & $38(2.5)$ \\
Above 50,000 & $6(1.9)$ & $9(0.7)$ & $8(0.5)$ \\
Total & $322(100.0)$ & $1,355(100.0)$ & $1,549(100.0)$ \\
\hline
\end{tabular}

Values are presented as number (\%).

FM: Family Medicine, JFP: Journal of Family Practice, KJFM: Korean Journal of Family Medicine. 
had the largest size 2,846,210 subjects of a US NHANES based a cross-sectional study in 2005. The largest data size of KJFM was $6,072,196$ subjects in registry study which used health insurance information in 1997, and a cancer prevention data-based cohort study was $1,306,476$ subjects in 2006 .

\section{Research Design}

In all three journals, the majority of the articles were crosssectional studies, with $72.4 \%$ in KJFM, 58.4\% in JFP, and $66.8 \%$ in FM. Among the prospective studies, cohort studies were $0.8 \%$ in KJFM, 3.1\% in JFP, and 5.0\% in FM and the clinical trials were $6.3 \%$ in KJFM, $12.6 \%$ in JFP, and $15.2 \%$ in FM. Among retrospective studies, historical cohort studies were $0.3 \%$ in KJFM, 1.5\% in JFP, and 1.9\% in FM and case-control studies were $8.6 \%$ in $\mathrm{KJFM}, 10.2 \%$ in JFP, and $0.6 \%$ in $\mathrm{FM}$, moreover registry studies were $11.6 \%$ in KJFM, $14.2 \%$ in JFP, and $10.6 \%$ in FM (Table 5).

The proportions of prospective studies and retrospective studies in JFP were higher than those in KJFM until 2002, and KJFM showed higher proportions of prospective studies and retrospective studies than those of JFP from 2003 (Table 6).

\section{Statistical Methods}

From 1978 to 1987, articles which used only descriptive

Table 5. Classification of original article by research design.

\begin{tabular}{lcccc}
\hline \multicolumn{1}{c}{ Research design } & FM & JFP & KJFM & Total \\
\hline Cross-sectional & $215(66.8)$ & $791(58.4)$ & $1,121(72.4)$ & $2,127(65.9)$ \\
Prospective & & & & \\
$\quad$ Cohort & $16(5.0)$ & $42(3.1)$ & $12(0.8)$ & $70(2.2)$ \\
$\quad$ Clinical trial & $49(15.2)$ & $171(12.6)$ & $98(6.3)$ & $318(9.9)$ \\
Retrospective & & & $5(0.3)$ & $32(1.0)$ \\
$\quad$ Historical cohort & $6(1.9)$ & $21(1.5)$ & $133(8.6)$ & $273(8.5)$ \\
$\quad$ Case-control & $2(0.6)$ & $138(10.2)$ & $180(11.6)$ & $406(12.6)$ \\
Registry & $34(10.6)$ & $192(14.2)$ & $1,549(100.0)$ & $3,226(100.0)$ \\
Total & $322(100.0)$ & $1,355(100.0)$ & & \\
\hline
\end{tabular}

Values are presented as number (\%).

FM: Family Medicine, JFP: Journal of Family Practice, KJFM: Korean Journal of Family Medicine.

Table 6. Classification of yearly research design by three journals.

\begin{tabular}{|c|c|c|c|c|c|c|c|c|c|c|c|c|}
\hline \multirow{2}{*}{ Years } & \multicolumn{3}{|c|}{ Cross-sectional } & \multicolumn{3}{|c|}{ Prospective } & \multicolumn{3}{|c|}{ Retrospective } & \multicolumn{3}{|c|}{ Total } \\
\hline & FM & JFP & KJFM & FM & JFP & KJFM & FM & JFP & KJFM & FM & JFP & KJFM \\
\hline 1978-1982 & - & $122(9.0)$ & $1(0.1)$ & - & $15(1.1)$ & $0(0.0)$ & - & $20(1.5)$ & $0(0.0)$ & $0(0.0)$ & 157 (11.6) & $1(0.1)$ \\
\hline $1983-1987$ & - & $328(24.2)$ & $21(1.4)$ & - & $59(4.4)$ & $1(0.1)$ & - & $111(8.2)$ & $8(0.5)$ & $0(0.0)$ & 498 (36.8) & $30(1.9)$ \\
\hline 1988-1992 & - & 216 (15.9) & 144 (9.3) & - & $76(5.6)$ & $10(0.6)$ & - & $101(7.5)$ & $58(3.7)$ & $0(0.0)$ & 393 (29.0) & $212(13.7)$ \\
\hline $1993-1997$ & - & $90(6.6)$ & 250 (16.1) & - & $31(2.3)$ & $14(0.9)$ & - & $80(5.9)$ & $81(5.2)$ & $0(0.0)$ & 201 (14.8) & 345 (22.3) \\
\hline 1998-2002 & $73(22.7)$ & $17(1.3)$ & $281(18.1)$ & $26(8.1)$ & $23(1.7)$ & $24(1.5)$ & $15(4.7)$ & $24(1.8)$ & $98(6.3)$ & 114 (35.4) & $64(4.7)$ & 403 (26.0) \\
\hline $2003-2007$ & $96(29.8)$ & $13(1.0)$ & 223 (14.4) & $21(6.5)$ & $5(0.4)$ & $29(1.9)$ & $19(5.9)$ & $12(0.9)$ & $57(3.7)$ & 136 (42.2) & $30(2.2)$ & 309 (19.9) \\
\hline $2008-2011$ & 46 (14.3) & $5(0.4)$ & $201(13.0)$ & $18(5.6)$ & $4(0.3)$ & $32(2.1)$ & $8(2.5)$ & $3(0.2)$ & $16(1.0)$ & $72(22.4)$ & $12(0.9)$ & 249 (16.1) \\
\hline Total & 215 (66.8) & 791 (58.4) & 1,121 (72.4) & 65 (20.2) & 213 (15.7) & $110(7.1)$ & $42(13.0)$ & 351 (25.9) & $318(20.5)$ & $322(100.0)$ & 1,355 & 1,549 (100.0) \\
\hline
\end{tabular}

Values are presented as number (\%).

FM: Family Medicine, JFP: Journal of Family Practice, KJFM: Korean Journal of Family Medicine. 


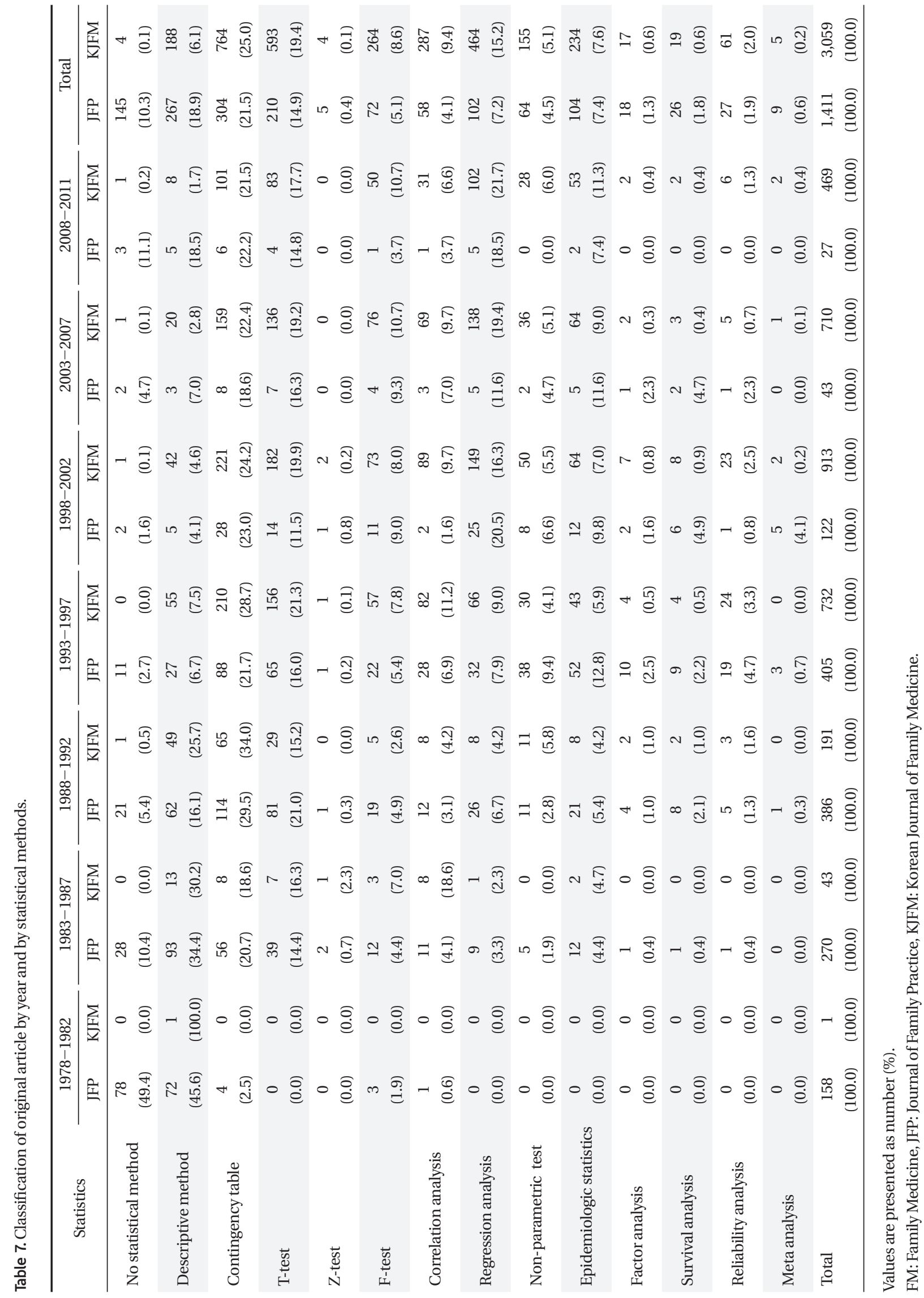


methods were $34.4 \%$ in KJFM and $30.2 \%$ in JFP. However, from 1988 to 2011, contingency table and t-test were the most frequently used in KJFM and JFP, approximately $25 \%$ and $15 \%$. The articles using reliability analysis in KJFM increased from 1.6\% in 1992 to $3.3 \%$ in 1993, whereas JFP increased from $1.3 \%$ in 1992 to $4.7 \%$ in 1993 (Table 7 ).

\section{New Terms and Methods}

A ridit analysis for comparing ordinal scale responses such as degree of injury, dissatisfaction, and preference or agreement, was used by KJFM in 1999. The Breslow-Day test to examine the homogeneity of odds ratios, was used for the first time in 2002, and a cost-effectiveness analysis was first reported in an article related to health service in 2004.

In 1978, risk-benefit analysis for the comparison between the risk and its related benefits was mentioned in an article related to health service in JFP. In 2002, articles that used Cochran's Q test, which assesses the homogeneity or consistency within the group, and funnel plot in a meta-analysis were used for the first time.

In FM, a path analysis which used to find the causal relationship between variables, was reported in 1999, and a content analysis which categorizes the contents subjectively in volume to analyze them based on certain standards, was used for the first time in 2009.

\section{DISCUSSION}

As a result of comparing original articles published by KJFM 1,549, JFP 1,355, and FM 322, KJFM had a small number of papers, smaller data sizes, and relatively simple statistical methods until 1992 compared to FM and JFP. However, the proportions of original articles published by KJFM increased from 1.9\% in 1987 to $13.7 \%$ in 1988, and observational studies of KJFM has increased since 1997.

KJFM and JFP were $61.7 \%$ and $67.2 \%$ in the medical area, and $\mathrm{FM}$ was $55.9 \%$ in the education area. Yu et al. ${ }^{2)}$ analyzed articles published by KJFM and JFP from 1981 to 1992, and found that KJFM had $51.1 \%$ articles and JFP had $44.4 \%$ in the biomedical area. These results showed similar as this study on the fact that both KJFM and JFP had the highest portion of articles in the biomedical area.
In all three journals, the majority of the articles used data sizes of $100-300$ subjects, which were $29.1 \%$ in KJFM, $26.1 \%$ in JFP, and $28.6 \%$ in FM. Also, FM showed the highest proportion, 1.9\%, of articles that used data sizes over 50,000 subjects.

In research design, cross-sectional studies were mostly used in all three journals; $72.4 \%$ in KJFM, 58.4\% in JFP, and 66.8\% in FM. FM had the highest proportion, $20.2 \%$, of prospective studies, and JFP showed the highest proportion, 25.9\%, of retrospective studies. Jacobsen and Meininger ${ }^{5)}$ analyzed articles published in nursing research from 1956 to 1983 and found that the proportion of cross-sectional studies was larger than that of longitudinal studies. This result showed the same result as this study in the fact that both KJFM and JFP had the highest portion of cross-sectional studies.

Contingency table and t-test were the most frequently used in KJFM, with proportions of $25 \%$ and $19.4 \%$, regression analysis $15.2 \%$, correlation analysis $9.4 \%$, and F-test $8.6 \%$ were used. Moreover, complex statistical methods such as epidemiologic statistics, survival analysis, and reliability analysis have been used increasingly since 1993. Sim et al. ${ }^{6)}$ analyzed the original articles published in KJFM, and found that chi-square, t-test and analysis of variance (ANOVA) were the most frequently used, also, Lee and $\mathrm{Ahn}^{7)}$ found that $30.6 \%$ of the medical articles published in Korea in the 1980s did not even mention their statistical methods, and that most articles used t-test, correlation analysis, and contingency table. Although there could be some differences of categorization in regards to the statistical methods, it showed that the statistical methods such as chi-square test, t-test, ANOVA, and correlation analysis were highly used in many medical articles.

In conclusion, it was confirmed that the study activities in the field of family medicine have become more active over the years, but still has conducted based on the relatively small data sizes and weak research designs. It will be helpful to conduct prospective studies such as cohort studies and clinical trials, also, we need to collaborate to accumulate the small sized data sets and try to make a registry. More refined statistical method such as a propensity score matching analysis for retrospective data could be an alternative to increase the academic level of family medicine. 


\section{CONFLICT OF INTEREST}

No potential conflict of interest relevant to this article was reported.

\section{REFERENCES}

1. Fromm BS, Snyder VL. Research design and statistical procedures used in the Journal of Family Practice. J Fam Pract 1986;23:564-6.

2. Yu BS, Yun MY, Hong SH, Jo JY. Analysis of the article published in the Journal of the Korean Academy of Family Medicine and the Journal of Family Practice: from January 1981 to December 1992. J Korean Acad Fam Med 1994;15:251-64.
3. Seo HG, Hwang IH, Huh BY. The analysis of the articles published in the Journal of the Korean Academy of Family Medicine: from November 1980 to June 1989.J Korean Acad Fam Med 1990;11:8-16.

4. Shank JC. A taxonomy for research. Fam Med Teach $1980 ; 12: 22-3$.

5. Jacobsen BS, Meininger JC. The designs and methods of published nursing research: 1956-1983. Nurs Res 1985;34:306-12.

6. Sim KW, Lee KJ, Lee ES, Park WM, Lee SH, Lee HS, et al. Analysis of papers published in Journal of the Korean Academy of Family Medicine. J Korean Acad Fam Med 1998;19:353-63.

7. Lee HK, Ahn YO. An assessment of methodological and statistical validity of medical articles published in Korea, from 1980 to 1989. Korean J Med Educ 1991;3:52-69. 